\title{
Some Mathematical Properties of the Dynamically Inconsistent Bellman Equation: A Note on the Two-Sided Altruism Dynamics
}

\author{
Aoki Takaaki \\ Institute of Economics Research, Kyoto University
}

Received: September 27, 2014 / Accepted: October 21, 2014 / Published: January 25, 2015.

\begin{abstract}
This article describes some dynamic aspects on dynastic utility incorporating two-sided altruism with an OLG setting. The special case is analyzed where the weights of two-sided altruism are dynamically inconsistent. The Bellman equation for two-sided altruism proves to be reduced to one-sided dynamic problem, but the effective discount factor is different only in the current generation. It is shown that a contraction mapping result of value function cannot be achieved in general, and that there can locally exist an infinite number of self-consistent policy functions of the class $C^{n}$ with distinct steady states (indeterminacy of self-consistent, differentiable policy functions).
\end{abstract}

Keywords: Bellman equation, Two-sided altruism, Dynamic inconsistency, Self-consistent policy functions, Indeterminacy, Overlapping generations model.

\section{Introduction}

This paper analyzes some mathematical aspects of two sided altruism dynamics especially under dynamic inconsistency, with constant fertility and no saving. The model is based on so-called Buiter-Carmichael-Burbidge (BCB) type two-sided utility, which we modify for the three stage OLG model, so that each generation might hold, in general, two chances of intergenerational linkage, firstly

I acknowledge the financial support of the Grant-in-Aid for Scientific Research, JSPS (\#23000001), "Complex Dynamic Analysis on Economic Crisis and Social Infrastructure". I am grateful to Dr. Toshihiro Iwai and Dr. Jose-Victor Rios-Rull for comments. Of course, all errors are my own. This paper was presented at the Mathematical Society of Japan, Annual Meeting, on March 17, 2014, in Tokyo, Japan, at SIAM Conference on Optimization 14, CP 21 Optimal Control IV, on May 21, 2014, in San-Diego, U.S.A., at Asian Meeting of the Econometric Society (AMES 2014), on June 21, 2014, in Taipei, the Republic of China (Taiwan), and at 14th SAET Conference on Current Trends in Economics, on August 21, 2014, in Tokyo, Japan.

Corresponding author: Aoki Takaaki, Kyoto University, Institute of Economic Research, Yoshida-honmachi, Sakyo-ku, Kyoto, 606-8501, Japan, Phone: +81-75-753-7184. E-mail: aoki@kier.kyoto-u.ac.jp through fertility and capital investment decision planned by middle age parent during young adulthood, and secondly through transfer (compensation/bequest) during old stage. As explained later, this modification proves to induce some interesting, but puzzling behaviors in macro-dynamics, especially under dynamic inconsistency.

As references, two-sided altruism dynamics are treated, for example, in Abel (1987), Kimball (1987), Hori and Kanaya (1989), Altig and Davis (1993), Hori (1997), Aoki (2011). Furthermore, the differentiability of value functions is discussed in Benveniste and Scheinkman (1979), Santos (1991), Araujo (1991), Montrucchio (1987). Mathematical treatments regarding the principle of optimality appear, for example, in Bellman (1957), Pontryagin (1962), Blackwell (1965), Stokey and Lucas (1989), and Mitra (2000). Boldrin and Montrucchio (1986), and Geanakoplos and Brown (1985) are located at the earlier stage among the "indeterminacy of equilibrium" literature. Furthermore, Krusell P., Kuruşçu B. and Smith A. A. 
(2002), and Krusell P. and Smith A. A. (2003, 2008) analyze the quasi-geometric discounting model.

The organization of this paper is as follows. We describe the model in section 2, and theoretical results in section 3 , and finally concluding remarks in section 4 .

\section{Model}

We assume a typical OLG model consisting of three life stages, $C, Y, O$ (childhood, young adulthood (working age) and old adulthood (retirement stage)). Generation $t$, who spends its young adulthood (stage $Y$ ) at period $t$, shares the adjacent life stages with generation $t+1$ and $t-1$. (For example, stage $O$ of old parents and stage $Y$ of young children are shared simultaneously.) The whole life utility of generation $t$ is defined as $u_{t}=u_{t}^{(y)}+\delta u_{t+1}^{(o)}$, where $u_{t}^{(y)}$ and $u_{t+1}^{(o)}$ are the young and old adulthood utility of generation $t$, respectively, and $\delta$ is a time preference discount factor for old (retirement) stage. At period $t$, generation $\mathrm{t}$ decides some of its life strategies, fertility $\left(n_{t}\right)$ and capital investment for children $\left(k_{t+1}\right)$ and saving for forthcoming retirement stage $O\left(s_{t}\right)$, and gift for old parents $\left(b_{t}\right)$. Just for simplicity, we assume that fertility is constant $\left(n_{t}=1\right)$ and there is no saving $\left(s_{t}=0\right)$, and that only the gift for old parents is controllable.

\subsection{Representative Agent Problem}

Now we consider the following type of two sided altruism, where $u_{t}$ and $V_{t}$ and is an individual life utility and a (two-sided) dynastic utility of generation $t$, respectively.

$$
\begin{gathered}
V_{t}=\alpha \delta u_{t}^{(o)}+\sum_{s=0}^{\infty} \beta^{s} u_{t+s}=\alpha \delta u_{t}^{(o)}+\sum_{s=0}^{\infty} \beta^{s}\left(u_{t+s}^{(y)}+\delta u_{t+1+s}^{(o)}\right)= \\
\alpha \delta u_{t}^{(o)}+\left(u_{t}^{(y)}+\delta u_{t+1}^{(o)}\right)+\beta\left(u_{t+1}^{(y)}+\delta u_{t+2}^{(o)}\right)+\beta^{2}\left(u_{t+2}^{(y)}+\delta u_{t+3}^{(o)}\right)+\cdots .
\end{gathered}
$$

This representation is an OLG version of Buiter-Carmichael-Burbidge (BCG) type utility of the form, $V_{t}=\alpha u_{t-1}+u_{t}+\sum_{s=1}^{\infty} \beta^{s} u_{t+s}$. (As for the BCG utility, see Abel (1987)). We assume $\beta<1$.

Now denote consumptions at stage $Y$ (period $t$ ) and $O$ (period $t+1)$ of generation $t$, by $c_{1, t}$ and $c_{2, t+1}$, respectively. Then $c_{1, t}=f\left(k_{t}\right)-k_{t+1}-b_{t}$ and $c_{2, t+1}=b_{t+1}$, where $f(\cdot)$ is a production function, $k_{t}$ is a human capital of generation $t$ with a full depreciation in one period, and $b_{t+1}$ is a gift from young adult generation $t+1$ to old adult generation $t$. Assuming the intertemporally separable utility form, $u(c)=c^{1-\sigma} /(1-\sigma)$ for $\sigma \neq 1$, and $\ln c$ for $\sigma=1$, we have $u_{t}^{(y)}=u\left(c_{1, t}\right)$ and $u_{t+1}^{(o)}=u\left(c_{2, t+1}\right)$.

If all the period $t$ and subsequent strategies $\left\{b_{t^{\prime}}, k_{t^{\prime}+1}\right\}_{t^{\prime}=t}^{\infty}$ are independently determined by generation $t$, then the generation solves the following representative agent problem.

$$
\max _{\left\{k_{t^{\prime}+1}, b_{t^{\prime}}\right\}^{\infty}=t} V_{t}
$$

However, in this OLG linkage, the inconsistent motive for intergenerational transfer between young children and old parents makes each generation behave differently from RA1. To see why, we rewrite $V_{t}$ as

$$
\begin{aligned}
V_{t} & =\left(u_{t}^{(y)}+\alpha \delta u_{t}^{(o)}\right)+\beta\left(u_{t+1}^{(y)}+(\delta / \beta) u_{t+1}^{(o)}\right)+\beta^{2}\left(u_{t+2}^{(y)}+(\delta / \beta) u_{t+2}^{(o)}\right)+\cdots \\
& =\left(u_{t}^{(y)}+\alpha \delta u_{t}^{(o)}\right)+\sum_{s=1}^{\infty} \beta^{s}\left(u_{t+s}^{(y)}+(\delta / \beta) u_{t+s}^{(o)}\right) .
\end{aligned}
$$

Maximizing $V_{t}$ in RA1 necessarily assures that the ratio of marginal utility in consumption between young and old adults be $1: \alpha \delta$ at period $t$, while $1: \delta / \beta$ at period $t^{\prime} \quad\left(t^{\prime}=t+1, t+2, \cdots\right)$. As a 
matter of fact, however, all generations $t^{\prime}(\geq t)$ are to adjust their gifts and allocate their consumptions with old parents by $1: \alpha \delta$ in the ratio of marginal utility. Therefore, the corresponding representative agent problem should be rather the following sequential problem.

$$
\max _{\left\{k_{t^{\prime}+1}, \hat{b}_{t^{\prime}}\right\}_{t^{\prime}=t}^{\infty}} V_{t}
$$

s.t. $\hat{b}_{t^{\prime}}=b_{t^{\prime}} \operatorname{argmax}\left\{u_{t^{\prime}}^{(y)}+\alpha \delta u_{t^{\prime}}^{(o)}\right\} \quad$ given $k_{t^{\prime}}$ and $k_{t^{\prime}+1}, t^{\prime}=t, t+1, t+2, \cdots$.

A solution of the constraint, $\hat{b}_{t^{\prime}}$ $\left(t^{\prime}=t, t+1, t+2, \cdots\right)$, can be derived explicitly as $\hat{b}_{t^{\prime}}=\frac{1}{1+(\alpha \delta)^{-1 / \sigma}}\left\{f\left(k_{t^{\prime}}\right)-k_{t^{\prime}+1}\right\} \quad, \quad$ so $\quad$ defining $\hat{c}_{1, t^{\prime}} \equiv f\left(k_{t^{\prime}}\right)-k_{t^{\prime}+1}-\hat{b}_{t^{\prime}}=\frac{1}{1+(\alpha \delta)^{1 / \sigma}}\left\{f\left(k_{t^{\prime}}\right)-k_{t^{\prime}+1}\right\}$ and $\hat{c}_{2, t^{\prime}} \equiv \hat{b}_{t^{\prime}}=\frac{(\alpha \delta)^{1 / \sigma}}{1+(\alpha \delta)^{1 / \sigma}}\left\{f\left(k_{t^{\prime}}\right)-k_{t^{\prime}+1}\right\}$, we have $u\left(\hat{c}_{1, t^{\prime}}\right)+\alpha \delta u\left(\hat{c}_{2, t^{\prime}}\right) \equiv A u\left(\tilde{C}_{t^{\prime}}\right)$ and $u\left(\hat{c}_{1, t^{\prime}}\right)+(\delta / \beta) u\left(\hat{c}_{2, t^{\prime}}\right) \equiv B u\left(\tilde{C}_{t^{\prime}}\right) \quad, \quad$ where $A \equiv\left\{1+(\alpha \delta)^{1 / \sigma}\right\}^{\sigma} \quad, \quad B \equiv \frac{\left\{1+(\alpha \delta)^{1 / \sigma}(\alpha \beta)^{-1}\right\}}{\left\{1+(\alpha \delta)^{1 / \sigma}\right\}^{1-\sigma}}, \quad$ and $\tilde{C}_{t^{\prime}} \equiv f\left(k_{t^{\prime}}\right)-k_{t^{\prime}+1}$.

Thus defining $\breve{V}_{t}$, which internalizes the old age support by each generation according to the $1: \alpha \delta$ rule, $\hat{b}_{t^{\prime}}=b_{t^{\prime}} \operatorname{argmax}\left\{u_{t^{\prime}}^{(y)}+\alpha \delta u_{t^{\prime}}^{(o)}\right\}$,

$$
\begin{gathered}
\breve{V}_{t}=A u\left(\tilde{C}_{t}\right)+\beta B u\left(\tilde{C}_{t+1}\right)+\beta^{2} B u\left(\tilde{C}_{t+2}\right)+ \\
\beta^{3} B u\left(\tilde{C}_{t+3}\right)+\cdots=A\left\{u\left(\tilde{C}_{t}\right)+\beta \mu u\left(\tilde{C}_{t+1}\right)\right. \\
\left.+\beta^{2} \mu u\left(\tilde{C}_{t+2}\right)+\beta^{3} \mu u\left(\tilde{C}_{t+3}\right)+\cdots\right\},
\end{gathered}
$$

where $\mu=B / A$. Here $\quad \mu=1$ if $\alpha \beta=1$ (dynamically consistent). Thus the effective discount factor is $\beta \mu$ at the present period $t$, but $\beta$ from the next period $t+1$. Therefore the objective function proves to hold a sort of the quasi-geometric discounting time structure, as discussed in Krusell et al. (2002, 2003 and 2008).

Finally RA2 can be simply rewritten as

$$
\max _{\left\{k_{t^{\prime}+1}^{\infty}\right\}_{t^{\prime}=t}^{\infty}} \breve{V}_{t^{\prime}} \text {. }
$$

It is obvious that RA2' or RA2 are equivalent with
RA1 if and only if $\mu=1$ (i.e., $\alpha \beta=1)$. We call this case $(\mu=1)$ dynamic consistency, and otherwise inconsistency. From time consistency requirement $\alpha=\frac{1-\sqrt{1-4 a b}}{2 b}, \quad \beta=\frac{1-\sqrt{1-4 a b}}{2 a} \quad(a b \leq 1 / 4)$, we have $\alpha \beta<1$, therefore $\mu>1$, that is, the model is dynamically inconsistent. See Kimball (1987), Hori et al. (1989) and Hori (1997).

\subsection{Functional Bellman Equation}

Now we try to rewrite the representative agent problem represented in RA2', in the form of recursive functional Bellman equation.

At first, we define another objective function $\tilde{V}_{t}$,

$$
\begin{gathered}
\tilde{V}_{t}=B u\left(\tilde{C}_{t}\right)+\beta B u\left(\tilde{C}_{t+1}\right)+\beta^{2} B u\left(\tilde{C}_{t+2}\right)+ \\
\beta^{3} B u\left(\tilde{C}_{t+3}\right)+\cdots=B\left\{u\left(\tilde{C}_{t}\right)+\beta u\left(\tilde{C}_{t+1}\right)+\right. \\
\left.\beta^{2} u\left(\tilde{C}_{t+2}\right)+\beta^{3} u\left(\tilde{C}_{t+3}\right)+\cdots\right\},
\end{gathered}
$$

where

$$
\tilde{V}_{t}=\breve{V}_{t}+(B-A) u\left(\tilde{C}_{t}\right) .
$$

Then two sided altruism dynamics is described as the following one sided functional equation, where dual value functions, $\breve{W}_{t}(\cdot)$ and $\tilde{W}_{t}(\cdot)$, correspond with objective functions $\breve{V}_{t}$ and $\tilde{V}_{t}$, respectively.

$$
\begin{aligned}
& \breve{W}_{t}\left(k_{t}\right)= \\
& \max _{0 \leq k_{t+1} \leq f\left(k_{t}\right)}\left(A u\left(f\left(k_{t}\right)-k_{t+1}\right)+\beta \tilde{W}_{t+1}\left(k_{t+1}\right)\right),
\end{aligned}
$$

where

$$
\tilde{W}_{t}\left(k_{t}\right)=\breve{W}_{t}\left(k_{t}\right)+(B-A) u\left(\hat{C}_{t}\right)
$$

and $\hat{C}_{t}=f\left(k_{t}\right)-\hat{k}_{t+1}$,

where

$$
\hat{k}_{t+1}=\underset{0 \leq k_{t+1} \leq f\left(k_{t}\right)}{\arg \max }\left(A u\left(f\left(k_{t}\right)-k_{t+1}\right)+\beta \tilde{W}_{t+1}\left(k_{t+1}\right)\right) .
$$

$\hat{k}_{t+1}=g_{t}\left(k_{t}\right)$ is a policy function of generation $t$, given next generation $t+1$ 's value functions $\breve{W}_{t+1}(\cdot)$ and $\tilde{W}_{t+1}(\cdot)$. BE1 is a simpler version of two sided altruism model examined by Hori (1997), eqs. (4.2)-(4.6). 
Under dynamic inconsistency $(\mu \neq 1)$, the maximization problem of representative agent's utility (RA2' or RA2) is not equivalent with the corresponding Bellman equation (BE1) with the same objective function $\breve{V}_{t}$, even though some transversality conditions are appropriately assumed. As a matter of fact, there exist two effects caused by dynamic inconsistency: A. intra-temporal direct effect on intergenerational transfer, and B. inter-temporal indirect effect on preceding generations' policy functions. And Bellman equation proves to internalize both effects $\mathrm{A}$ and $\mathrm{B}$, while representative agent utility maximization does only effect $A$. In addition, the principle of optimality does not hold in general under dynamic inconsistency, so that Blackwell's contraction mapping theorem (Stokey \& Lucas (1989) Theorem 3.3) cannot be applied. However, this complete internalization induces some perplexing aspects in BE1, which does not appear in case of dynamic consistency.

To see this, we now rewrite BE1 in a backwardly recursive fashion.

$$
\breve{W}_{n+1}(k)=\max _{y \in Y(k)}\left(A u(f(k)-y)+\beta \tilde{W}_{n}(y)\right),
$$

where

$$
\tilde{W}_{n+1}(k)=\breve{W}_{n+1}(k)+(B-A) u(\hat{C}) .
$$

$n$ is a time distance from the future terminal period $n=0$. Here $\hat{C}=f(k)-\hat{y}$, where $\hat{y}=\arg \max \left(A u(f(k)-y)+\beta \tilde{W}_{n}(y)\right) \equiv g_{n+1}(k)$ $Y(k)$ is a feasible correspondence defined as $Y(k)=\{y \mid 0 \leq y \leq f(k)\}$.

In case of dynamic consistency $(\mu=B / A=1)$, the above equation reduces to a regular Bellman equation,

$$
W_{n+1}(k)=\max _{y \in Y(k)}\left(A u(f(k)-y)+\beta W_{n}(y)\right),
$$

where $W_{n}(\cdot)=\tilde{W}_{n}(\cdot)=\breve{W}_{n}(\cdot)$.

Then, under quite general conditions, the principle of optimality is known to assure a uniform convergence of $W_{n}(\cdot)$ to time-independent value function $W(\cdot)$, which satisfies

$$
W(k)=\max _{y \in Y(k)}(A u(f(k)-y)+\beta W(y)) .
$$

In case of dynamic inconsistency $(\mu \neq 1)$, such a contraction mapping result, for example, by Blackwell (1965), cannot be automatically expected. However roughly dare to consider, at any events, the following time-independent functional equation.

$$
\breve{W}(k)=\max _{y \in Y(k)}(A u(f(k)-y)+\beta \tilde{W}(y)),
$$

where

$$
\tilde{W}(k)=\breve{W}(k)+(B-A) u(\hat{C}),
$$

and

$$
\hat{C}=f(k)-\hat{y}
$$
and

$\hat{y}=\underset{y \in Y(k)}{\arg \max }(A u(f(k)-y)+\beta \tilde{W}(y)) \equiv g(k)$.

So far we assume a priori the existence of policy function $g_{n}(k)$ instead of policy correspondence, implicitly its differentiability, the uniform convergence of convergence of $g_{n}(k)$ to $g(k)$, and so on. See, for example, Stokey et al. (1989). Some of these conditions prove to hold even under dynamic inconsistency, but some do not. In the next section, we will investigate the analytical properties of BE2 and BE1' from various viewpoints.

\section{Results}

Let be $R_{+}=\{x \in R \mid x \geq 0\}$ and define $K \subset R_{+}$, the domain of capital $k$, so that $k \in K$. Also assume that $f: R_{+} \rightarrow R_{+}$and $u: R_{+} \rightarrow R_{+}$are differentiable and satisfy the following properties:

Assumptions:

F0: Production function $f(k)$ is $C^{\infty}$, i.e., infinitely continuously differentiable.

F1: $f(0)=0$.

F2: $f$ is strictly concave.

F3: $f^{\prime}(k)>0$.

F4: $\lim _{k \rightarrow 0} f^{\prime}(k)=\infty, \lim _{k \rightarrow \infty} f^{\prime}(k)=0$. 
U0: Utility function $u(c)$ is $C^{\infty}$, i.e., infinitely continuously differentiable. Specifically $u(c)=c^{1-\sigma} /(1-\sigma)$ for $\sigma \neq 1$, where $\sigma$ is a relative risk aversion or an inverse of elasticity of intertemporal substitution.

$\mathrm{U} 1: u(0)=0$.

$\mathrm{U} 2: u$ is strictly concave.

U3: $u^{\prime}(c)>0$ for $c>0$.

U4: $\lim _{c \rightarrow 0} u^{\prime}(c)=\infty, \lim _{c \rightarrow \infty} u^{\prime}(c)=0$.

Theorem 1 derives a modified Euler equation corresponding with two-sided altruism.

Theorem 1: In BE2 assume that value function $\tilde{W}(k)$ and policy function $\hat{y}=g(k)$ are $C^{1}$, i.e., once continuously differentiable, and that $\hat{y}=g(k)$ is an interior of the feasible correspondence $Y(k)$. Then BE2 satisfies the following Euler equation EE1, modified for case of dynamic inconsistency.

$$
\begin{aligned}
& -u^{\prime}(f(k)-y)+ \\
& \beta u^{\prime}(f(y)-g(y))\left[\mu f^{\prime}(y)-(\mu-1) g^{\prime}(y)\right]=0,
\end{aligned}
$$

where $y=\hat{g}(k)$ is a solution of EE1.

Proof is trivial and left for Appendix. EE1 (Euler equation under dynamic inconsistency) is equivalent with the "generalized Euler equation", as derived in Krusell et al. (2002). This is plausible, because the OLG model under dynamic inconsistency proves to be equivalent with a sort of the quasi-geometric discounting model. Then time consistency requires that it happens to be $\hat{g}(k)=g(k)$. Therefore EE1 can be rewritten as

$$
\begin{aligned}
& -u^{\prime}((f-g)(k))+\beta u^{\prime}((f-g) \circ g(k)) \times \\
& {\left[\left(\mu f^{\prime}-(\mu-1) g^{\prime}\right) \circ g(k)\right]=0,}
\end{aligned}
$$

where $a \circ b(x) \equiv a(b(x))$ denotes a composite function of $x$, and $(a \pm b)(x) \equiv a(x) \pm b(x)$.

If $g(k)$ satisfies EE2, then we say $g(k)$ is self-consistent, in the sense that if next generation's policy function is $g(k)$, then the current generation necessarily takes the same policy.
At a fixed point, $k=k^{*}, g\left(k^{*}\right)=k^{*}$ and $-u^{\prime}\left(c^{*}\right)+\beta u^{\prime}\left(c^{*}\right)\left[\mu f^{\prime}\left(k^{*}\right)-(\mu-1) g^{\prime}\left(k^{*}\right)\right]=0$, where $\quad c^{*}=f\left(k^{*}\right)-k^{*} \quad$. Thus $\beta\left[\mu f^{\prime}\left(k^{*}\right)-(\mu-1) g^{\prime}\left(k^{*}\right)\right]=1 \quad(\star)$ holds. Note that $\beta f^{\prime}\left(k^{*}\right)=1$ implies $g^{\prime}\left(k^{*}\right)=1 / \beta$, and that $\beta \mu f^{\prime}\left(k^{*}\right)=1$ implies $g^{\prime}\left(k^{*}\right)=0$.

\subsection{Indeterminacy of self-consistent policy functions}

Next theorem relates dynamic inconsistency with indeterminacy of self-consistent policy functions.

Theorem 2: Assume $\mu \neq 1$. Let $\bar{k}$ be a point such that $\beta \mu f^{\prime}(\bar{k})=1$, and take any point such that $k^{*} \neq \bar{k}$. Then, there exists uniquely a self-consistent policy function $g(k)$ satisfying EE2, such that it has a fixed point at $k=k^{*}$, and is $C^{n}$, i.e., infinitely continuously differentiable, for any positive integer $n$ on some open ball around $k^{*}, k \in B\left(k^{*}, \varepsilon\right)$, i.e., $k^{*}-\varepsilon<k<k^{*}+\varepsilon$, with $\varepsilon>0$.

Proof is given in Appendix. This theorem says that if a fixed point $k^{*}$ is determined, then a corresponding self-consistent policy function is also uniquely determined and $C^{n}$ in an open ball around $k^{*}$. Since there exists a trade-off and degree of freedom between the values of $g\left(k^{*}\right)$ and $g^{\prime}\left(k^{*}\right)$, it is possible to construct an infinite number of distinct self-consistent policy functions for distinct $k^{*}$. While Krusell et al. (2003) find out the indeterminacy of saving rules (policy functions), which is a step function, non-differentiable at and converging to some arbitrary stationary point, this theorem provides a rigorous proof that an infinite number of distinct $C^{n}$ self-consistent policy functions exist with distinct stationary points.

\subsection{Existence of $\mathrm{C}^{n}$ Self-Consistent Value} Functions with Uniform Convergence

Given a self-consistent policy function $g(k)$ satisfying EE2, self-consistent value functions $\tilde{Z}(k)$ and $\breve{Z}(k)$ in duality, which correspond with $\tilde{W}(k)$ and $\breve{W}(k)$, respectively, are defined as the following infinite functional series. 


$$
\begin{gathered}
\tilde{Z}(k)=B\{u((f-g)(k))+ \\
\beta u(f-g) \circ g(k))+ \\
\left.\beta^{2} u((f-g) \circ g \circ g(k))+\cdots\right\} \\
=B \sum_{s=0}^{\infty} \beta^{s} u((f-g) s \circ \underbrace{g \circ \cdots \circ g}_{s}(k)) \\
=B \sum_{s=0}^{\infty} \beta^{s} u\left((f-g) \circ g_{(s)}(k)\right)
\end{gathered}
$$

and

$$
\breve{Z}(k)=\tilde{Z}(k)-(B-A) u((f-g)(k)) .
$$

Here we define $g_{(s)}(k) \equiv s \underbrace{g \circ \cdots \circ g}_{s}(k)$. It is easy to verify $\tilde{Z}\left(k^{*}\right)=(B /(1-\beta)) u\left(f\left(k^{*}\right)-k^{*}\right)$. Now we claim the following theorem.

Theorem 3: Assume $\mu \neq 1$. Let $S$ be a set such that

$$
S=\left\{k||\left(\mu f^{\prime}(k)-1 / \beta\right) /(\mu-1) \mid<1\right\},
$$

and take any point such that $k^{*} \in S$ and $k^{*} \neq \bar{k}$. Then:

(i) Self-consistent value functions $\tilde{Z}(k)$ and $\breve{Z}(k)$, which are represented as an infinite series SV1, where $g(k)$ satisfies EE2, uniformly converges and once continuously differentiable $C^{1}$ on some open ball around $k^{*}, k \in B\left(k^{*}, \varepsilon^{\prime}\right)$, with $\varepsilon^{\prime}>0$.

(ii) In $\mathrm{BE} 2$, replace $\tilde{W}(\cdot)$ and $\breve{W}(\cdot)$ with $\mathrm{y}$ and $\breve{Z}(\cdot)$, respectively. Then $\tilde{Z}(\cdot)$ and $\breve{Z}(\cdot)$ satisfy BE2 with a unique self-consistent policy function $g(k)$.

(iii) Let $S^{\prime}$ be a set such that $S^{\prime}=\left\{k \mid 0<\left(\mu f^{\prime}(k)-1 / \beta\right) /(\mu-1)<1\right\}$, and take any point such that $k^{*} \in S^{\prime}$, instead of $S$. Then $\tilde{Z}(k)$ is strictly concave at $k^{*}$.

Proof is given in Appendix. By the proof $\tilde{Z}(k)$ and $\breve{Z}(k)$ are also shown to be $C^{n}$. Thus BE2 is now formally justified as the following Bellman equation.

$$
\breve{Z}(k)=\max _{y \in Y(k)}(A u(f(k)-y)+\beta \tilde{Z}(y)),
$$

where

$$
\begin{gathered}
\tilde{Z}(k)=\breve{Z}(k)+(B-A) u(\hat{C}), \\
\text { and } \quad \hat{C}=f(k)-\hat{y} \\
\hat{y}=y \in \underset{Y(k)}{\arg \max }(A u(f(k)-y)+\beta \tilde{Z}(y)) .
\end{gathered}
$$

Theorem 3 says that $\hat{y}$ in BE3 must be the same as $g(k)$, if c and $\breve{Z}(k)$ are defined as SV1.

\subsection{Instability Against Perturbation of Self-Consistent Policy/Value Functions}

In section 3.2, at first we searched out the policy functions, which satisfies Euler equation EE2 locally around a fixed point $k^{*}$, and then calculate the corresponding value functions. Therefore it is not still verified if deviated policy functions would necessarily converges to some of self-consistent ones in a global sense. So we go back to a recursive Bellman equation BE1'. At the terminal stage $n=0$,

$$
\breve{W}_{1}(k)=\max _{y \in Y(k)}\left(A u(f(k)-y)+\beta \tilde{W}_{0}(y)\right),
$$

where

$$
\tilde{W}_{1}(k)=\breve{W}_{1}(k)+(B-A) u(\hat{C}) .
$$

Assume a $\log$ utility $u(c)=\ln c$ and a Cobb-Douglus form production function $f(k)=a k^{b}$, although this utility does not satisfy condition U1. Assuming $\tilde{W}_{0}(k)=0 \quad$ (therefore $\left.g_{0}(k)=0\right)$, it is easy to verify, by recursive calculation, that $g_{n}(k)=\gamma_{n} f(k)$, where $\gamma_{n}=\frac{\beta \mu b\left\{1-(\beta b)^{n}\right\}}{(1-\beta b)+\beta \mu b\left\{1-(\beta b)^{n}\right\}} \quad$ and $g_{n}(k) \underset{k \rightarrow \infty}{\rightarrow} g(k)=\gamma f(k)$, where $\quad \gamma=\frac{\beta \mu b}{1-\beta b(1-\mu)}$. $(g(k)=\gamma f(k) \quad$ satisfies self-consistent Euler equation EE2.) As a matter of fact, if $g_{0}(k)$ belongs to a family of functions of a Cobb-Douglus form, $g_{0}(k)=\gamma_{0} f(k) \quad\left(0 \leq \gamma_{0}<1\right)$, then it is proved that $g_{n}(k) \underset{k \rightarrow \infty}{\rightarrow} g(k)=\gamma f(k)$, the same destination function. However, in general, every possible initial policy function $g_{0}(k)$ might not necessarily attain a 
uniform convergence to $g(k)$. This point is totally different from case of dynamic consistency $\mu=1$. (See Blackwell (1965) for a contraction result in case of $\mu=1$.)

Here we limit our focus on the local stability against temporal perturbation of self-consistent policy and value functions.

Theorem 4: Let $h(k)$ be a $C^{\infty}$ function, which is bounded in an open ball around $k^{*}$, and $h\left(k^{*}\right) \neq 0$. Also let $g(k)$ be a self-consistent policy function, which satisfies EE2, and let $\tilde{Z}(k)$ be a corresponding self-consistent value function generated by SV1. Assume both $g(k)$ and $\tilde{Z}(k)$ are $C^{n}$ in an open ball around $k^{*}$.

(i) Assume that the next generation's policy function is subject to a perturbation of the form: $g(y) \rightarrow \tilde{g}(y, \eta)=g(y)+\eta h(y)$, and the current generation's policy function changes $g(k) \rightarrow \hat{g}(k, \eta)$.

Then the condition for the policy function's contraction in an open neighborhood around $k^{*}$, $k \in B\left(k^{*}, \varepsilon^{\prime \prime}\right)$ with some $\varepsilon^{\prime \prime}>0$, is

$$
\left|\frac{g^{\prime}\left(k^{*}\right)}{f^{\prime}\left(k^{*}\right)}\left\{1+\beta(\mu-1) \frac{h^{\prime}\left(k^{*}\right)}{h\left(k^{*}\right)} \frac{u^{\prime}\left(c^{*}\right)}{u^{\prime \prime}\left(c^{*}\right)}\right\}\right|<1 \text {. }
$$

(ii) Assume that the next generation's value function is subject to a perturbation of the form: $\tilde{Z}(y) \rightarrow \tilde{Z}(y, \eta)=\tilde{Z}(y)+\eta h(y)$, and the current generation's value function changes $\tilde{Z}(k) \rightarrow \hat{Z}(k, \eta)$.
Then the condition for the value functions's contraction in an open neighborhood around $k^{*}$, $k \in B\left(k^{*}, \varepsilon^{\prime \prime}\right)$ with some $\varepsilon^{\prime \prime}>0$, is

$$
\left|\beta\left\{1+(\mu-1) \frac{h^{\prime}\left(k^{*}\right)}{h\left(k^{*}\right)} \frac{g^{\prime}\left(k^{*}\right)}{f^{\prime}\left(k^{*}\right)} \frac{u^{\prime}\left(c^{*}\right)}{u^{\prime \prime}\left(c^{*}\right)}\right\}\right|<1 .
$$

Proof is given in Appendix. Both results (i) and (ii) are similar. In case of dynamic consistency $\mu=1$, the contraction can be achieved under quite general conditions, $\quad\left|g^{\prime}\left(k^{*}\right) / f^{\prime}\left(k^{*}\right)\right|<1$ or $\beta<1$, in which the local convergence in sup norm $\left\|g_{n}-g\right\|_{K} \underset{n \rightarrow \infty}{\rightarrow} 0$ or $\left\|\tilde{Z}_{n}-\tilde{Z}\right\|_{K} \underset{n \rightarrow \infty}{\rightarrow} 0$ are attained, whatever the first order or the higher orders of perturbation $\left(h^{\prime}(k), h^{\prime \prime}(k), h^{\prime \prime \prime}(k) \cdot \cdot\right)$ might be. However, in case of $\mu \neq 1$, the first order perturbation $h^{\prime}(k)$ or the first order nondifferentiability directly affects the possibility of $0^{\prime}$ th order contraction (in sup norm), and so do the second or higher perturbation $\left(h^{\prime \prime}(k), h^{\prime \prime \prime}(k), \cdot \cdot\right)$, or the nondifferentiability in these orders, indirectly. So finally in the next theorem we state the first order effect on $\hat{g}(k, \eta)$ of perturbation $h(k)$ around $k^{*}$, which is measured by $\hat{g}_{12}\left(k^{*}, 0\right)$.

Theorem 5: Under the same assumptions as in (i) of Theorem 4, the $\hat{g}_{12}\left(k^{*}, 0\right)$, the first order effect of $\hat{g}\left(k^{*}, \eta\right)$ for a small change in $\eta h(y)$, is given in the following formula.

$$
\hat{g}_{12}\left(k^{*}, 0\right)=X_{0} h\left(k^{*}\right)+X_{1} h^{\prime}\left(k^{*}\right)+X_{2} h^{\prime \prime}\left(k^{*}\right),
$$

Where

$$
\begin{gathered}
X_{0}=-\frac{g^{\prime}\left(k^{*}\right)}{f^{\prime}\left(k^{*}\right)}\left(\frac{g^{\prime}\left(k^{*}\right)}{f^{\prime}\left(k^{*}\right)}\left\{f^{\prime \prime}\left(k^{*}\right) g^{\prime}\left(k^{*}\right)-f^{\prime}\left(k^{*}\right) g^{\prime \prime}\left(k^{*}\right)\right\}-\frac{u^{\prime \prime}\left(c^{*}\right)}{u^{\prime}\left(c^{*}\right)}\left\{f^{\prime}\left(k^{*}\right)-g^{\prime}\left(k^{*}\right)\right\}\left\{1-g^{\prime}\left(k^{*}\right)\right\}\right), \\
X_{1}=\frac{g^{\prime}\left(k^{*}\right)}{f^{\prime}\left(k^{*}\right)}\left(\begin{array} { c } 
{ g ^ { \prime } ( k ^ { * } ) \{ f ^ { \prime } ( k ^ { * } ) - g ^ { \prime } ( k ^ { * } ) \} } \\
{ g ^ { \prime } ( k ^ { * } ) + \beta ( \mu - 1 ) }
\end{array} \left[\begin{array}{c}
\left.\left.-\frac{u^{\prime}\left(c^{*}\right)}{u^{\prime \prime}\left(c^{*}\right)} \frac{g^{\prime}\left(k^{*}\right)}{f^{\prime}\left(k^{*}\right)}\left(\begin{array}{c}
\left\{f^{\prime \prime}\left(k^{*}\right) g^{\prime}\left(k^{*}\right)-f^{\prime}\left(k^{*}\right) g^{\prime \prime}\left(k^{*}\right)\right\} \\
+\frac{u^{\prime \prime \prime}\left(c^{*}\right)}{u^{\prime \prime}\left(c^{*}\right)} \frac{g^{\prime}\left(k^{*}\right)}{f^{\prime}\left(k^{*}\right)}\left\{f^{\prime}\left(k^{*}\right)-g^{\prime}\left(k^{*}\right)\right\}
\end{array}\right)\right]\right),
\end{array}\right.\right.
\end{gathered}
$$




$$
X_{2}=\beta(\mu-1) \frac{\left\{g^{\prime}\left(k^{*}\right)\right\}^{2}}{f^{\prime}\left(k^{*}\right)} \frac{u^{\prime}\left(c^{*}\right)}{u^{\prime \prime}\left(c^{*}\right)} .
$$

Proof is given in Appendix. Let $\eta h_{n}(k)$ denote a functional deviation from self-consistent policy function $g(k)$ at stage $n$. In case of dynamic consistency $\quad \mu=1$, then $X_{1}=\frac{\left\{g^{\prime}\left(k^{*}\right)\right\}^{2}}{f^{\prime}\left(k^{*}\right)}$ and $X_{2}=0$. From Theorem 4, $h_{n}\left(k^{*}\right) \rightarrow 0$, as $n \rightarrow \infty$. Since $\frac{\left\{g^{\prime}\left(k^{*}\right)\right\}^{2}}{f^{\prime}\left(k^{*}\right)}<1, \quad h_{n}^{\prime}\left(k^{*}\right)$ proves to converge to zero. That is, qualitatively speaking, the order-by-order derivative contraction operates in general. However, in case of dynamic inconsistency $\mu \neq 1$, the higher order derivative coefficient affects the lower one, and the lower one, if failing in contraction, remains an obstacle for contraction in the higher one, as $n \rightarrow \infty$.

\section{Concluding Remarks}

Thus this paper describes a dynamics of one-sector growth model under two sided altruism. Here we derived a modified Euler equation for dynamic inconsistency.

From viewpoints of macrodynamics \& game theory, one important implication of this paper is that even under this perfect foresight setting with a perfectly rational representative agent (in the sense that each generation takes account of and internalizes all the predictable reaction by the subsequent generations), dynamic inconsistency still induces indeterminacy of self-consistent policy functions, and possibly cause some dynamic fluctuation of policy function generated in recursive fashion.

This aspect is crucial not only in this two-sided altruism dynamics, but also in other models incorporating irregular structures of variable effective discount factors, as in hyperbolic discount factor model, endogenized (so variable) discount factor model, or fertility endogenized model.

This paper focused on self-consistency, differentiability, and fragility against recursive perturbation of policy/value functions, in a local area around any arbitrary fixed point $k^{*}$. Investigation on global transition in $\mathrm{BE} 1$ or $\mathrm{BE} 1$ ', characterized by dynamic fluctuation, will be left for future work.

\section{References}

[1] Abel A. B. (1987) "Operative Gift and Bequest Motives", American Economic Review, 77/5, 1037-1047.

[2] Aoki T. (2011) "On the Implications of Two-Sided Altruism in Human Capital Based OLG Model", Asian Journal of Law and Economics, 2/2.

[3] Araujo A. (1991) "The once but not twice differentiability of the policy function", Econometrica, 59/ 5, 1383-1393.

[4] Bellman R. (1957) Dynamic Programming, Princeton, Princeton University Press.

[5] Benveniste, L. M. and Scheinkman J. A. (1979) "On the Differentiability of the Value Function in Dynamic Models of Economics", Econometrica, 47/3, 727-732.

[6] Blackwell D. (1965) "Discounted Dynamic Programming”, Annals of Mathematical Statistics, 36, 226-235.

[7] Boldrin M. and Montrucchio L. (1986) "On the Indeterminacy of Capital Accumulation Paths", Journal of Economic Theory, 40/1, 26-39.

[8] Geanakoplos J. and Brown D. J. (1985) "Comaparative Statics and Local Indeterminacy in OLG Economics: An Application of the Maultiplicative Ergodic Theorem", Cowles Foundation Discussion Paper, 773.

[9] Hori H. (1997) "Dynamic Allocation in an Altruistic Overlapping Generations Economy", Journal of Economic Theory, 73/2, 292-315.

[10] Hori H. and Kanaya S. (1989) "Utility Functionals with Nonpaternalistic Intergenerational Altruism", Journal of Economic Theory, 49, 241-265.

[11] Kimball M. S. (1987) "Making Sense of Two-Sided Altruism", Journal of Monetary Economics, 20/2, 301-326.

[12] Krusell P., Kuruşçu B. and Smith A. A. (2002) "Equiribrium Welfare and Governmental Policy with Quasi-geometric Discounting”, Journal of Economic Theory, 105, 42-72.

[13] Krusell P. and Smith A. A. (2003) "Consumption-savings Decisions with Quasi-geometric Discounting", Econometrica, 71-1, 365-375.

[14] Krusell P. and Smith A. A. (2008) "Consumption-savings Decisions with Quasi-geometric Discounting: The Case with a Discrete Domain”, Working Paper. 
A Note on the Two-sided Altruism Dynamics

[15] Mitra T. (2000) "Introduction to Dynamic Optimization Theory", Optimization and Chaos, Majumdar, Mukal, Tapan Mitra and Kazuo Nishimura eds, Studies in Economic Theory, Springer.

[16] Montrucchio L. (1987) "Lipschiz continuous policy functions for strongly concave optimization problems", Journal of Mathematical Economics, 16, 259-273.
[17] Pontryagin, L. S. and Associates (1962) The Mathematical Theory of Optimal Processes, New York: Interscience.

[18] Santos M. S. (1991) "Smoothness of the Policy Function in Discrete Time Economic Models", Econometrica, 59/5, 1365-1382.

[19] Stokey N. and Lucas R. E. (1989) Recursive Methods in Economic Dynamics, Harvard University Press. 


\section{Appendix}

\section{Proof of Theorem 1}

From the assumption, $\hat{y}$ is an interior of $Y(k)$. Differentiating BE2 with $k$, we have $-A u^{\prime}(f(k)-\hat{y})+\beta \tilde{W}^{\prime}(\hat{y})=0$ (A ). Then, since $\hat{y}=g(k)$ and $g(k)$ is once continuously differentiable,

$$
\begin{aligned}
\breve{W}^{\prime}(k) & =A u^{\prime}(f(k)-\hat{y}) f^{\prime}(k)+\left(\frac{d \hat{y}}{d k}\right) \underbrace{\left[-A u^{\prime}(f(k)-\hat{y})+\beta \tilde{W}^{\prime}(\hat{y})\right]}_{=0} \\
= & A u^{\prime}(f(k)-g(k)) f^{\prime}(k) .
\end{aligned}
$$

Then, from $\tilde{W}(k)=\breve{W}(k)+(B-A) u(f(k)-g(k))$,

$$
\begin{aligned}
\tilde{W}^{\prime}(k) & =A u^{\prime}(f(k)-g(k)) f^{\prime}(k)+(B-A) u^{\prime}(f(k)-g(k))\left\{f^{\prime}(k)-g^{\prime}(k)\right\} \\
& =u^{\prime}(f(k)-g(k))\left[B f^{\prime}(k)-(B-A) g^{\prime}(k)\right] \\
& =A u^{\prime}(f(k)-g(k))\left[\mu f^{\prime}(k)-(\mu-1) g^{\prime}(k)\right] .
\end{aligned}
$$

Plugging this into (A),

$$
-A u^{\prime}(f(k)-y)+\beta A u^{\prime}(f(y)-g(y))\left[\mu f^{\prime}(y)-(\mu-1) g^{\prime}(y)\right]=0 \text {. Divide this by } A \text {, finally we get EE1. }
$$

\section{Proof of Theorem 2}

At a fixed point $g\left(k^{*}\right)=k^{*}, \beta\left[\mu f^{\prime}\left(k^{*}\right)-(\mu-1) g^{\prime}\left(k^{*}\right)\right]=1$ holds. In case of $\mu \neq 1$, there exist an infinite number of combination of $g\left(k^{*}\right)$ and $g^{\prime}\left(k^{*}\right)$. Take any arbitrary point such that $k^{*} \neq \bar{k}$. Then $g^{\prime}\left(k^{*}\right) \neq 0$, and since $g(k)$ is $C^{1}$, there exists an open neighborhood around $k^{*}, B\left(k^{*}, \varepsilon\right)$, such that $\varepsilon$ is enough small, and $g^{\prime}(k)>0$ or $g^{\prime}(k)<0$ for all $k \in B\left(k^{*}, \varepsilon\right) . u(\cdot)$ and $f(\cdot)$ are $C^{\infty}$, then applying the implicit function theorem to EE2, $g^{\prime}(k)$ is $C^{1}$ on $B\left(k^{*}, \varepsilon\right)$ (that is, $g(k)$ is $C^{2}$ (twice continuously differentiable)). Differentiating EE2 with $k$, we get

$$
\begin{aligned}
& -u^{\prime \prime}(f(k)-g(k))\left\{f^{\prime}(k)-g^{\prime}(k)\right\} \\
& +\beta\left\{\begin{array}{c}
u^{\prime \prime}(f(y)-g(y))\left[\mu f^{\prime}(y)-(\mu-1) g^{\prime}(y)\right]\left\{f^{\prime}(y)-g^{\prime}(y)\right\} \\
+u^{\prime}(f(y)-g(y))\left[\mu f^{\prime \prime}(y)-(\mu-1) g^{\prime \prime}(y)\right]
\end{array}\right\} g^{\prime}(k) \\
& =0 .
\end{aligned}
$$

Here $y=g(k)$. Then at a fixed point $k^{*}$ with $c^{*}=f\left(k^{*}\right)-k^{*}$,

$$
\begin{aligned}
& -u^{\prime \prime}\left(c^{*}\right)\left\{f^{\prime}\left(k^{*}\right)-g^{\prime}\left(k^{*}\right)\right\} \\
& +\beta\left\{\begin{array}{c}
u^{\prime \prime}\left(c^{*}\right)\left[\mu f^{\prime}\left(k^{*}\right)-(\mu-1) g^{\prime}\left(k^{*}\right)\right]\left\{f^{\prime}\left(k^{*}\right)-g^{\prime}\left(k^{*}\right)\right\} \\
+u^{\prime}\left(c^{*}\right)\left[\mu f^{\prime \prime}\left(k^{*}\right)-(\mu-1) g^{\prime \prime}\left(k^{*}\right)\right]
\end{array}\right\} g^{\prime}\left(k^{*}\right) \\
& =0 .
\end{aligned}
$$

Arranging this with $\beta\left[\mu f^{\prime}\left(k^{*}\right)-(\mu-1) g^{\prime}\left(k^{*}\right)\right]=1(\star)$, we have the following equality. 


$$
\left\{1-g^{\prime}\left(k^{*}\right)\right\} u^{\prime \prime}\left(c^{*}\right)\left\{f^{\prime}\left(k^{*}\right)-g^{\prime}\left(k^{*}\right)\right\}=\beta g^{\prime}\left(k^{*}\right) u^{\prime}\left(c^{*}\right)\left[\mu f^{\prime \prime}\left(k^{*}\right)-(\mu-1) g^{\prime \prime}\left(k^{*}\right)\right]
$$

Thus, considering $\mu \neq 1, u^{\prime}\left(c^{*}\right) \neq 0$ and $g^{\prime}\left(k^{*}\right) \neq 0, g^{\prime \prime}\left(k^{*}\right)$ is uniquely determined.

Again applying the implicit function theorem to EE2-2, $g^{\prime \prime}(k)$ is $C^{1}$ on $B\left(k^{*}, \varepsilon\right)$ (that is, $g(k)$ is $C^{3}$ (three times continuously differentiable). Differentiating EE2-2 with $k$, and setting at a fixed point $k^{*}$,

$$
\begin{gathered}
-u^{\prime \prime}\left(c^{*}\right)\left\{f^{\prime \prime}\left(k^{*}\right)-g^{\prime \prime}\left(k^{*}\right)\right\}-u^{\prime \prime \prime}\left(c^{*}\right)\left\{f^{\prime}\left(k^{*}\right)-g^{\prime}\left(k^{*}\right)\right\}^{2} \\
\beta\left\{\begin{array}{c}
u^{\prime \prime \prime}\left(c^{*}\right)\left[\mu f^{\prime}\left(k^{*}\right)-(\mu-1) g^{\prime}\left(k^{*}\right)\right]\left\{f^{\prime}\left(k^{*}\right)-g^{\prime}\left(k^{*}\right)\right\}^{2} \\
+2 u^{\prime \prime}\left(c^{*}\right)\left[\mu f^{\prime \prime}\left(k^{*}\right)-(\mu-1) g^{\prime \prime}\left(k^{*}\right)\right]\left\{f^{\prime}\left(k^{*}\right)-g^{\prime}\left(k^{*}\right)\right\} \\
+u^{\prime \prime}\left(c^{*}\right)\left[\mu f^{\prime}\left(k^{*}\right)-(\mu-1) g^{\prime}\left(k^{*}\right)\right]\left\{f^{\prime \prime}\left(k^{*}\right)-g^{\prime \prime}\left(k^{*}\right)\right\} \\
+u^{\prime}\left(c^{*}\right)\left[\mu f^{\prime \prime \prime}\left(k^{*}\right)-(\mu-1) g^{\prime \prime \prime}\left(k^{*}\right)\right]
\end{array}\right\}\left\{g^{\prime}\left(k^{*}\right)\right\}^{2} \\
+\beta\left\{\begin{array}{c}
u^{\prime \prime}\left(c^{*}\right)\left[\mu f^{\prime}\left(k^{*}\right)-(\mu-1) g^{\prime}\left(k^{*}\right)\right]\left\{f^{\prime}\left(k^{*}\right)-g^{\prime}\left(k^{*}\right)\right\} \\
+u^{\prime}\left(c^{*}\right)\left[\mu f^{\prime \prime}\left(k^{*}\right)-(\mu-1) g^{\prime \prime}\left(k^{*}\right)\right]
\end{array}\right\} g^{\prime \prime}\left(k^{*}\right) \\
=0 .
\end{gathered}
$$

Similarly, $g^{\prime \prime \prime}\left(k^{*}\right)$ is uniquely determined. Thus, by induction, $g(k)$ is $C^{n}$ for any positive integer $n$, and the $n$ 'th order coefficient of derivative at $k^{*}, g^{(n)}\left(k^{*}\right)$, say, is uniquely determined at any positive integer $n$.

\section{Proof of Theorem 3}

(i) It is easy to verify that $k^{*} \neq \bar{k}$ and $k^{*} \in S$ imply $g^{\prime}\left(k^{*}\right) \neq 0$ and $\left|g^{\prime}\left(k^{*}\right)\right|<1$. Then there exists an open neighborhood around $k^{*}, B\left(k^{*}, \varepsilon^{\prime}\right)$, such that $\varepsilon^{\prime}$ is enough small, and $1>g^{\prime}(k)>0$ or $-1<g^{\prime}(k)<0$ for all $k \in B\left(k^{*}, \varepsilon^{\prime}\right)$. Then obviously,

$$
g_{(s)}\left(B\left(k^{*}, \varepsilon^{\prime}\right)\right) \subset g_{(s-1)}\left(B\left(k^{*}, \varepsilon^{\prime}\right)\right) \subset \cdots \subset g\left(B\left(k^{*}, \varepsilon^{\prime}\right)\right) \subset B\left(k^{*}, \varepsilon^{\prime}\right) .
$$

Since $u((f-g)(k))$ is positive and upper bounded on $B\left(k^{*}, \varepsilon^{\prime}\right)$, it holds that $\left\|u\left((f-g) \circ g_{(s)}(k)\right)\right\|_{B\left(k^{*}, \varepsilon^{\prime}\right)} \leq M_{0}$ for all $s \geq 0$, where $\|f\|_{K} \equiv \sup _{k \in K}|f(k)|$. In addition, $\sum_{s=0}^{\infty} \beta^{s} M_{0}$ converges, therefore, from Weierstrass's $\mathrm{M}$ test, an infinite functional series $B \sum_{s=0}^{\infty} \beta^{s} u\left((f-g) \circ g_{(s)}(k)\right)$ uniformly converges to $\tilde{Z}(k)$ on $B\left(k^{*}, \varepsilon^{\prime}\right)$. Each term $\beta^{s} u\left((f-g) \circ g_{(s)}(k)\right)$ is continuous on $B\left(k^{*}, \varepsilon^{\prime}\right)$, so is $\tilde{Z}(k)$ on $B\left(k^{*}, \varepsilon^{\prime}\right)$.

Next, differentiating $u\left((f-g) \circ g_{(s)}(k)\right)$ with $k$,

$$
\begin{aligned}
& \left(u\left((f-g) \circ g_{(s)}(k)\right)\right)^{\prime}=u^{\prime}\left((f-g) \circ g_{(s)}(k)\right)\left[\left(f^{\prime}-g^{\prime}\right) \circ\left(g_{(s)}(k)\right)\right]\left[g^{\prime}\left(g_{(s-1)}(k)\right)\right] \cdot \cdot\left[g^{\prime}(g(k))\right]\left[g^{\prime}(k)\right] \text {. } \\
& u\left((f-g) \circ g_{(s)}(k)\right) \text { is } C^{1} \text {, so }\left(u\left((f-g) \circ g_{(s)}(k)\right)\right)^{\prime} \quad \text { is continuous on } B\left(k^{*}, \varepsilon^{\prime}\right) \text {. Considering ( \& ), } \\
& \left\|u^{\prime}\left((f-g) \circ g_{(s)}(k)\right)\right\|_{B\left(k^{*}, \varepsilon^{\prime}\right)} \leq M_{1} \quad, \quad\left\|\left(f^{\prime}-g^{\prime}\right) \circ\left(g_{(s)}(k)\right)\right\|_{B\left(k^{*}, \varepsilon^{\prime}\right)} \leq M_{2} \quad \text { and } \quad\left\|g^{\prime}\left(g_{(u-1)}(k)\right)\right\|_{B\left(k^{*}, \varepsilon^{\prime}\right)} \leq 1
\end{aligned}
$$


$(0 \leq u \leq s)$. Therefore, $\left\|\left(u\left((f-g) \circ g_{(s)}(k)\right)\right)^{\prime}\right\|_{B\left(k^{*}, \varepsilon^{\prime}\right)} \leq M_{1} M_{2}$. Furthermore $\sum_{s=0}^{\infty} \beta^{s} M_{1} M_{2}$ converges, so $B \sum_{s=0}^{\infty} \beta^{s}\left(u\left((f-g) \circ g_{(s)}(k)\right)\right)^{\prime}$ uniformly converges and is continuous on $B\left(k^{*}, \varepsilon^{\prime}\right)$.

Summarizing the above, (1) $B \sum_{s=0}^{\infty} \beta^{s} u\left((f-g) \circ g_{(s)}(k)\right)$ converges to $\tilde{Z}(k),(2) u\left((f-g) \circ g_{(s)}(k)\right)$ is $C^{1}$, (3) $B \sum_{s=0}^{\infty} \beta^{s}\left(u\left((f-g) \circ g_{(s)}(k)\right)\right)^{\prime}$ uniformly converges. From (1), (2) and (3), the conditions for the term-by-term differentiability, $\tilde{Z}(k)$ is $C^{1}$, and $\tilde{Z}^{\prime}(k)=B \sum_{s=0}^{\infty} \beta^{s}\left(u\left((f-g) \circ g_{(s)}(k)\right)\right)^{\prime}$. The proof of the uniform convergence and $C^{1}$ (once differentiability) of $\breve{Z}(k)$ is now straightforward.

(ii) By the proof of (i), $\tilde{Z}^{\prime}(k)=B \sum_{s=0}^{\infty} \beta^{s}\left(u\left((f-g) \circ g_{(s)}(k)\right)\right)^{\prime}$. Then at a fixed point $k=k^{*}$,

$$
\begin{aligned}
\tilde{Z}^{\prime}\left(k^{*}\right) & =B \sum_{s=0}^{\infty}\left[\beta^{s} u^{\prime}\left(c^{*}\right) \cdot(f-g)^{\prime}\left(k^{*}\right) \cdot\left\{g^{\prime}\left(k^{*}\right)\right\}^{s}\right] \\
& =B u^{\prime}\left(c^{*}\right) \cdot(f-g)^{\prime}\left(k^{*}\right) \cdot \sum_{s=0}^{\infty}\left\{g^{\prime}\left(k^{*}\right)\right\}^{s} \\
& =B u^{\prime}\left(c^{*}\right) \cdot(f-g)^{\prime}\left(k^{*}\right) \cdot \frac{1}{1-g^{\prime}\left(k^{*}\right)},
\end{aligned}
$$

where $c^{*}=f\left(k^{*}\right)-k^{*} \cdot g(k)$ is, by definition, a solution of EE2 with a fixed point $g\left(k^{*}\right)=k^{*}$ and $(\star)$. Then

$$
\begin{aligned}
\beta\left[\mu f^{\prime}\left(k^{*}\right)-(\mu-1) g^{\prime}\left(k^{*}\right)\right]=1 & \Longleftrightarrow-A u^{\prime}\left(c^{*}\right)+\beta B u^{\prime}\left(c^{*}\right) \cdot(f-g)^{\prime}\left(k^{*}\right) \cdot \frac{1}{1-g^{\prime}\left(k^{*}\right)}=0 \\
& \Longleftrightarrow-A u^{\prime}\left(c^{*}\right)+\beta \tilde{Z}^{\prime}\left(k^{*}\right)=0
\end{aligned}
$$

Now define $g(k) \equiv \operatorname{argmax}_{y \in Y(k)}(A u(f(k)-y)+\beta \tilde{Z}(y))$. Then $g(k)$ is the only candidate solution of BE3. Since $g\left(k^{*}\right) \equiv \operatorname{argmax}_{y \in Y\left(k^{*}\right)}\left(A u\left(f\left(k^{*}\right)-y\right)+\beta \tilde{Z}(y)\right)$, and by $(\mathbf{\Delta})$, we have $g\left(k^{*}\right)=k^{*}$. If $g(k)$ is a solution of BE3, then by similar calculation as in Theorem 1, $g(k)$ proves to be a solution of EE2. Since $g(k)$ has a fixed point at $k^{*}$, by Theorem 2, $g(k)$ is uniquely determined at the neighborhood around $k^{*}$, therefore it must be that $g(k)=g(k)$ on $B\left(k^{*}, \varepsilon\right)$. It is obvious that if $g(k)=g(k)$, then $\breve{Z}(k)$, generated as $\tilde{Z}(k)=\breve{Z}(k)+(B-A) u(f(k)-g(k))$ in BE3, coincides with $\breve{Z}(k)$, as defined in SV1. Now we have proved that $\tilde{Z}(k)$ and $\breve{Z}(k)$ satisfy BE3 with a unique self-consistent policy function $g(k)$.

(iii) Next we prove a strict concavity of $\tilde{Z}(k)$ at $k=k^{*}$. From the proof of Theorem 1 , $\tilde{Z}^{\prime}(k)=\tilde{W}^{\prime}(k)=A u^{\prime}(f(k)-g(k))\left[\mu f^{\prime}(k)-(\mu-1) g^{\prime}(k)\right] \quad . \quad$ (It $\quad$ is $\quad$ easy to verify that $\tilde{Z}^{\prime}\left(k^{*}\right)=(A / \beta) u^{\prime}\left(c^{*}\right)>0$, where $c^{*}=f\left(k^{*}\right)-k^{*}$.) As $g(k)$ is $C^{n}$ on $B\left(k^{*}, \varepsilon^{\prime}\right)$, so $\tilde{Z}(k)$ and $\breve{Z}(k)$ are also $C^{n}$ on it. Again differentiating $\tilde{Z}^{\prime}(k)$ with $k$,

$$
\tilde{Z}^{\prime \prime}(k)=A\left[\begin{array}{c}
u^{\prime \prime}(f(k)-g(k))\left\{f^{\prime}(k)-g^{\prime}(k)\right\}\left\{\mu f^{\prime}(k)-(\mu-1) g^{\prime}(k)\right\} \\
+u^{\prime}(f(k)-g(k))\left\{\mu f^{\prime \prime}(k)-(\mu-1) g^{\prime \prime}(k)\right\}
\end{array}\right] .
$$


At $k=k^{*}$, using $(\star)$,

$$
\begin{aligned}
\tilde{Z}^{\prime \prime}\left(k^{*}\right) & =A\left[\begin{array}{c}
u^{\prime \prime}\left(c^{*}\right)\left\{f^{\prime}\left(k^{*}\right)-g^{\prime}\left(k^{*}\right)\right\}\left\{\mu f^{\prime}\left(k^{*}\right)-(\mu-1) g^{\prime}\left(k^{*}\right)\right\} \\
+u^{\prime}\left(c^{*}\right)\left\{\mu f^{\prime \prime}\left(k^{*}\right)-(\mu-1) g^{\prime \prime}\left(k^{*}\right)\right\}
\end{array}\right] \\
= & \frac{A}{\beta}\left[u^{\prime \prime}\left(c^{*}\right)\left\{f^{\prime}\left(k^{*}\right)-g^{\prime}\left(k^{*}\right)\right\}+\beta u^{\prime}\left(c^{*}\right)\left\{\mu f^{\prime \prime}\left(k^{*}\right)-(\mu-1) g^{\prime \prime}\left(k^{*}\right)\right\}\right] \\
= & \frac{A}{\beta}\left[u^{\prime \prime}\left(c^{*}\right)\left\{f^{\prime}\left(k^{*}\right)-g^{\prime}\left(k^{*}\right)\right\}+\frac{\left(1-g^{\prime}\left(k^{*}\right)\right)}{g^{\prime}\left(k^{*}\right)} u^{\prime \prime}\left(c^{*}\right)\left\{f^{\prime}\left(k^{*}\right)-g^{\prime}\left(k^{*}\right)\right\}\right] \\
= & \frac{A}{\beta g^{\prime}\left(k^{*}\right)} u^{\prime \prime}\left(c^{*}\right)\left\{f^{\prime}\left(k^{*}\right)-g^{\prime}\left(k^{*}\right)\right\} .
\end{aligned}
$$

Here we used the equality $(\bullet)$. From conditions U0 and U2, $u^{\prime \prime}\left(c^{*}\right)<0$. The assumption $k^{*} \in S^{\prime}$ assures $0<g^{\prime}\left(k^{*}\right)<1 / \beta$, which implies $f^{\prime}\left(k^{*}\right)-g^{\prime}\left(k^{*}\right)>0$. Therefore now we have $\tilde{Z}^{\prime \prime}\left(k^{*}\right)<0$, a desired result.

\section{Proof of Theorem 4}

(i) In EE1 replace $g(y)$ with $\tilde{g}(y, \eta)$, and $\hat{g}(k)$ with $\hat{g}(k, \eta)$, respectively, then we have:

$$
-u^{\prime}(f(k)-y)+\beta u^{\prime}(f(y)-\tilde{g}(y, \eta))\left[\mu f^{\prime}(y)-(\mu-1) \tilde{g}_{1}(y, \eta)\right]=0
$$

where $y=\hat{g}(k, \eta)$ and $\hat{g}(k, 0)=\tilde{g}(k, 0)=g(k)$.

Differentiating with $\eta$,

$$
\begin{aligned}
& u^{\prime \prime}(f(k)-y) \hat{g}_{2}(k, \eta) \\
& +\beta\left[\begin{array}{c}
-u^{\prime \prime}(f(y)-\tilde{g}(y, \eta))\left[\mu f^{\prime}(y)-(\mu-1) \tilde{g}_{1}(y, \eta)\right] \tilde{g}_{2}(y, \eta) \\
-u^{\prime}(f(y)-\tilde{g}(y, \eta))\left[(\mu-1) \tilde{g}_{12}(y, \eta)\right]
\end{array}\right. \\
& \left.+\left\{\begin{array}{c}
u^{\prime \prime}(f(y)-\tilde{g}(y, \eta))\left[\mu f^{\prime}(y)-(\mu-1) \tilde{g}_{1}(y, \eta)\right]\left\{f^{\prime}(y)-\tilde{g}_{1}(y, \eta)\right\} \\
+u^{\prime}(f(y)-\tilde{g}(y, \eta))\left[\mu f^{\prime \prime}(y)-(\mu-1) \tilde{g}_{11}(y, \eta)\right]
\end{array}\right\} \hat{g}_{2}(k, \eta)\right] \\
& =0 .
\end{aligned}
$$

Arranging this equation:

$$
\begin{aligned}
& \hat{g}_{2}(k, \eta)\left[\begin{array}{c}
u^{\prime \prime}(f(k)-y) \\
+\beta\left\{\begin{array}{c}
u^{\prime \prime}(f(y)-\tilde{g}(y, \eta))\left[\mu f^{\prime}(y)-(\mu-1) \tilde{g}_{1}(y, \eta)\right]\left\{f^{\prime}(y)-\tilde{g}_{1}(y, \eta)\right\} \\
+u^{\prime}(f(y)-\tilde{g}(y, \eta))\left[\mu f^{\prime \prime}(y)-(\mu-1) \tilde{g}_{11}(y, \eta)\right]
\end{array}\right\}
\end{array}\right] \\
& =\beta\left[\begin{array}{c}
u^{\prime \prime}(f(y)-\tilde{g}(y, \eta))\left[\mu f^{\prime}(y)-(\mu-1) \tilde{g}_{1}(y, \eta)\right] \tilde{g}_{2}(y, \eta) \\
+u^{\prime}(f(y)-\tilde{g}(y, \eta))\left[(\mu-1) \tilde{g}_{12}(y, \eta)\right]
\end{array}\right]
\end{aligned}
$$

Evaluating $\eta=0$ and $k=k^{*}\left(f\left(k^{*}\right)-k^{*}=c^{*}, k^{*}=g\left(k^{*}\right)\right)$, with $(\star)$ : 


$$
\begin{aligned}
& \hat{g}_{2}\left(k^{*}, 0\right)\left[\begin{array}{c}
u^{\prime \prime}\left(c^{*}\right)+u^{\prime \prime}\left(c^{*}\right)\left\{f^{\prime}\left(k^{*}\right)-g^{\prime}\left(k^{*}\right)\right\} \\
+\beta u^{\prime}\left(c^{*}\right)\left[\mu f^{\prime \prime}\left(k^{*}\right)-(\mu-1) g^{\prime \prime}\left(k^{*}\right)\right]
\end{array}\right] \\
& =u^{\prime \prime}\left(c^{*}\right) \tilde{g}_{2}\left(k^{*}, 0\right)+\beta(\mu-1) u^{\prime}\left(c^{*}\right) \tilde{g}_{12}\left(k^{*}, 0\right)
\end{aligned}
$$

From equality

$$
u^{\prime \prime}\left(c^{*}\right)+u^{\prime \prime}\left(c^{*}\right)\left\{f^{\prime}\left(k^{*}\right)-g^{\prime}\left(k^{*}\right)\right\}+\beta u^{\prime}\left(c^{*}\right)\left[\mu f^{\prime \prime}\left(k^{*}\right)-(\mu-1) g^{\prime \prime}\left(k^{*}\right)\right]=u^{\prime \prime}\left(c^{*}\right) \frac{f^{\prime}\left(k^{*}\right)}{g^{\prime}\left(k^{*}\right)} .
$$

So we obtain

$$
\hat{g}_{2}\left(k^{*}, 0\right)=\frac{g^{\prime}\left(k^{*}\right)}{f^{\prime}\left(k^{*}\right)}\left\{\tilde{g}_{2}\left(k^{*}, 0\right)+\beta(\mu-1) \frac{u^{\prime}\left(c^{*}\right)}{u^{\prime \prime}\left(c^{*}\right)} \tilde{g}_{12}\left(k^{*}, 0\right)\right\} .
$$

Evaluating at $k=k^{*}$ and $\eta=0$, with $\tilde{g}(k, \eta)=g(k)+\eta h(k), \tilde{g}_{2}\left(k^{*}, 0\right)=h\left(k^{*}\right)$ and $\tilde{g}_{12}\left(k^{*}, 0\right)=h^{\prime}\left(k^{*}\right)$,

$$
\frac{\hat{g}_{2}\left(k^{*}, 0\right)}{\tilde{g}_{2}\left(k^{*}, 0\right)}=\frac{g^{\prime}\left(k^{*}\right)}{f^{\prime}\left(k^{*}\right)}\left\{1+\beta(\mu-1) \frac{u^{\prime}\left(c^{*}\right)}{u^{\prime \prime}\left(c^{*}\right)} \frac{h^{\prime}\left(k^{*}\right)}{h\left(k^{*}\right)}\right\} .
$$

$\hat{g}_{2}\left(k^{*}, 0\right)$ and $\tilde{g}_{2}\left(k^{*}, 0\right)$ are the slopes of changes of the current and next generation's policy functions in a small change of $\eta$, evaluated at $k=k^{*}$ and $\eta=0$, respectively. So this is a desired result.

(ii) The F.O.C. (Euler eq.) of BE3 is $-A u^{\prime}(f(k)-y)+\beta \tilde{Z}^{\prime}(y)=0$. Replacing $y$ with $\hat{g}(k, \eta)$, and $\tilde{Z}(y)$ with $\tilde{Z}(y, \eta)$, then $-A u^{\prime}(f(k)-\hat{g}(k, \eta))+\beta \tilde{Z}_{1}(\hat{g}(k, \eta), \eta)=0 \quad(\sharp)$. Differentiating with $\eta$,

$$
A u^{\prime \prime}(f(k)-\hat{g}(k, \eta)) \hat{g}_{2}(k, \eta)+\beta\left(\tilde{Z}_{11}(\hat{g}(k, \eta), \eta) \hat{g}_{2}(k, \eta)+\tilde{Z}_{12}(\hat{g}(k, \eta), \eta)\right)=0 .
$$

Then we have

$$
\hat{g}_{2}(k, \eta)=\frac{-\beta \tilde{Z}_{12}(\hat{g}(k, \eta), \eta)}{A u^{\prime \prime}(f(k)-\hat{g}(k, \eta))+\beta \tilde{Z}_{11}(\hat{g}(k, \eta), \eta)}
$$

The current generation's value function $\hat{Z}(k, \eta)$ is calculated as $\hat{Z}(k, \eta)=B u(f(k)-\hat{g}(k, \eta))+\beta \tilde{Z}(\hat{g}(k, \eta), \eta)$. Then

$$
\begin{aligned}
\hat{Z}_{2}(k, \eta) & =-B u^{\prime}(f(k)-\hat{g}(k, \eta)) \hat{g}_{2}(k, \eta)+\beta\left(\tilde{Z}_{1}(\hat{g}(k, \eta), \eta) \hat{g}_{2}(k, \eta)+\tilde{Z}_{2}(\hat{g}(k, \eta), \eta)\right) \\
& =(A-B) u^{\prime}(f(k)-\hat{g}(k, \eta)) \hat{g}_{2}(k, \eta)+\beta \tilde{Z}_{2}(\hat{g}(k, \eta), \eta) .
\end{aligned}
$$

Here we used the equality $(\sharp)$. Also $\tilde{Z}_{2}(\hat{g}(k, \eta), \eta)=h(\hat{g}(k, \eta))$ and $\tilde{Z}_{12}(\hat{g}(k, \eta), \eta)=h^{\prime}(\hat{g}(k, \eta))$. Then plugging $(\dagger)$ into $(\dagger)$

$$
\hat{Z}_{2}(k, \eta)=\beta h(\hat{g}(k, \eta))\left\{1-\frac{h^{\prime}(\hat{g}(k, \eta))}{h(\hat{g}(k, \eta))} \cdot \frac{(A-B) u^{\prime}(f(k)-\hat{g}(k, \eta))}{A u^{\prime \prime}(f(k)-\hat{g}(k, \eta))+\beta \tilde{Z}_{11}(\hat{g}(k, \eta), \eta)}\right\} .
$$

Evaluating at $k=k^{*}$ and $\eta=0$, with $\hat{g}\left(k^{*}, 0\right)=g\left(k^{*}\right)=k^{*} \quad, \quad c^{*} \equiv f\left(k^{*}\right)-k^{*} \quad$ and 


$$
\begin{aligned}
\tilde{Z}_{11}\left(\hat{g}\left(k^{*}, 0\right), 0\right)= & \tilde{Z}^{\prime \prime}\left(k^{*}\right)= \\
\frac{\hat{Z}_{2}\left(k^{*}, 0\right)}{\beta g^{\prime}\left(k^{*}\right)} u^{\prime \prime}\left(c^{*}\right)\left\{f^{\prime}\left(k^{*}\right)-g^{\prime}\left(k^{*}\right)\right\}, & \beta\left\{1-\frac{h^{\prime}\left(k^{*}\right)}{h\left(k^{*}\right)} \cdot \frac{(A-B) u^{\prime}\left(c^{*}\right)}{A u^{\prime \prime}\left(c^{*}\right)+\frac{A}{g^{\prime}\left(k^{*}\right)} u^{\prime \prime}\left(c^{*}\right)\left\{f^{\prime}\left(k^{*}\right)-g^{\prime}\left(k^{*}\right)\right\}}\right\} \\
& =\beta\left\{1-\frac{h^{\prime}\left(k^{*}\right)}{h\left(k^{*}\right)} \cdot \frac{(1-\mu) u^{\prime}\left(c^{*}\right)}{u^{\prime \prime}\left(c^{*}\right)+\frac{1}{g^{\prime}\left(k^{*}\right)} u^{\prime \prime}\left(c^{*}\right)\left\{f^{\prime}\left(k^{*}\right)-g^{\prime}\left(k^{*}\right)\right\}}\right\} \\
& =\beta\left\{1+(\mu-1) \frac{h^{\prime}\left(k^{*}\right)}{h\left(k^{*}\right)} \frac{g^{\prime}\left(k^{*}\right)}{f^{\prime}\left(k^{*}\right)} \frac{u^{\prime}\left(c^{*}\right)}{u^{\prime \prime}\left(c^{*}\right)}\right\} .
\end{aligned}
$$

$\hat{Z}_{2}\left(k^{*}, 0\right)$ and $\tilde{Z}_{2}\left(k^{*}, 0\right)\left(=h\left(k^{*}\right)\right)$ are the slopes of changes of the current and next generation's value functions in a small change of $\eta$, evaluated at $k=k^{*}$ and $\eta=0$, respectively. This is also a desired result.

\section{Proof of Theorem 5}

Differentiating (@) with $k$,

$$
\begin{aligned}
& u^{\prime \prime}(f(k)-y) \hat{g}_{12}(k, \eta)+u^{\prime \prime \prime}(f(k)-y)\left\{f^{\prime}(k)-\hat{g}_{1}(k, \eta)\right\} \hat{g}_{2}(k, \eta) \\
& +\beta\left[\begin{array}{c}
\left\{\begin{array}{c}
-u^{\prime \prime \prime}(f(y)-\tilde{g}(y, \eta))\left\{f^{\prime}(k)-\hat{g}_{1}(k, \eta)\right\}\left[\mu f^{\prime}(y)-(\mu-1) \tilde{g}_{1}(y, \eta)\right] \\
-u^{\prime \prime}(f(y)-\tilde{g}(y, \eta))\left[\mu f^{\prime \prime}(y)-(\mu-1) \tilde{g}_{11}(y, \eta)\right] \\
+\left(\begin{array}{c}
-u^{\prime \prime}(f(y)-\tilde{g}(y, \eta))\left[\mu f^{\prime}(y)-(\mu-1) \tilde{g}_{1}(y, \eta)\right] \\
-(\mu-1) u^{\prime \prime}(f(y)-\tilde{g}(y, \eta))\left\{f^{\prime}(k)-\hat{g}_{1}(k, \eta)\right\}
\end{array}\right) \tilde{g}_{12}(y, \eta) \\
-u^{\prime}(f(y)-\tilde{g}(y, \eta))\left[(\mu-1) \tilde{g}_{112}(y, \eta)\right]
\end{array}\right\} \hat{g}_{1}(k, \eta) \\
+\left\{\begin{array}{c}
u^{\prime \prime \prime}(f(y)-\tilde{g}(y, \eta))\left[\mu f^{\prime}(y)-(\mu-1) \tilde{g}_{1}(y, \eta)\right]\left\{f^{\prime}(y)-\tilde{g}_{1}(y, \eta)\right\}^{2} \\
+2 u^{\prime \prime}(f(y)-\tilde{g}(y, \eta))\left[\mu f^{\prime \prime}(y)-(\mu-1) \tilde{g}_{11}(y, \eta)\right]\left\{f^{\prime}(y)-\tilde{g}_{1}(y, \eta)\right\} \\
+u^{\prime \prime}(f(y)-\tilde{g}(y, \eta))\left[\mu f^{\prime}(y)-(\mu-1) \tilde{g}_{1}(y, \eta)\right]\left\{f^{\prime \prime}(y)-\tilde{g}_{11}(y, \eta)\right\} \\
+u^{\prime}(f(y)-\tilde{g}(y, \eta))\left[\mu f^{\prime \prime \prime}(y)-(\mu-1) \tilde{g}_{111}(y, \eta)\right]
\end{array}\right\} \hat{g}_{1}(k, \eta) \hat{g}_{2}(k, \eta) \\
+\left\{\begin{array}{c}
u^{\prime \prime}(f(y)-\tilde{g}(y, \eta))\left[\mu f^{\prime}(y)-(\mu-1) \tilde{g}_{1}(y, \eta)\right]\left\{f^{\prime}(y)-\tilde{g}_{1}(y, \eta)\right\} \\
+u^{\prime}(f(y)-\tilde{g}(y, \eta))\left[\mu f^{\prime \prime}(y)-(\mu-1) \tilde{g}_{11}(y, \eta)\right]
\end{array}\right\} \hat{g}_{12}(k, \eta)
\end{array}\right]=0 .
\end{aligned}
$$

Evaluating $\eta=0$ and $k=k^{*}\left(f\left(k^{*}\right)-k^{*}=c^{*}, k^{*}=g\left(k^{*}\right)\right)$, 


$$
\begin{aligned}
& u^{\prime \prime}\left(c^{*}\right) \hat{g}_{12}\left(k^{*}, 0\right)+u^{\prime \prime \prime}\left(c^{*}\right)\left\{f^{\prime}\left(k^{*}\right)-g^{\prime}\left(k^{*}\right)\right\} \hat{g}_{2}\left(k^{*}, 0\right) \\
& +\beta\left[\left\{\left(\begin{array}{c}
-u^{\prime \prime \prime}\left(c^{*}\right)\left\{f^{\prime}\left(k^{*}\right)-g^{\prime}\left(k^{*}\right)\right\}\left[\mu f^{\prime}\left(k^{*}\right)-(\mu-1) g^{\prime}\left(k^{*}\right)\right] \\
-u^{\prime \prime}\left(c^{*}\right)\left[\mu f^{\prime \prime}\left(k^{*}\right)-(\mu-1) g^{\prime \prime}\left(k^{*}\right)\right] \\
+\left(\begin{array}{c}
-u^{\prime \prime}\left(c^{*}\right)\left[\mu f^{\prime}\left(k^{*}\right)-(\mu-1) g^{\prime}\left(k^{*}\right)\right] \\
-(\mu-1) u^{\prime \prime}\left(c^{*}\right)\left\{f^{\prime}\left(k^{*}\right)-g^{\prime}\left(k^{*}\right)\right\}
\end{array}\right) \tilde{g}_{12}\left(k^{*}, 0\right) \\
-u^{\prime}\left(c^{*}\right)\left[(\mu-1) \tilde{g}_{112}\left(k^{*}, 0\right)\right] \\
+S \cdot g^{\prime}\left(k^{*}\right) \cdot \hat{g}_{2}\left(k^{*}, 0\right) \\
+T \cdot \hat{g}_{12}\left(k^{*}, 0\right)
\end{array}\right]=0(\mathbf{O}),\right.\right.
\end{aligned}
$$

where

$$
S=\left\{\begin{array}{c}
u^{\prime \prime \prime}\left(c^{*}\right)\left[\mu f^{\prime}\left(k^{*}\right)-(\mu-1) g^{\prime}\left(k^{*}\right)\right]\left\{f^{\prime}\left(k^{*}\right)-g^{\prime}\left(k^{*}\right)\right\}^{2} \\
+2 u^{\prime \prime}\left(c^{*}\right)\left[\mu f^{\prime \prime}\left(k^{*}\right)-(\mu-1) g^{\prime \prime}\left(k^{*}\right)\right]\left\{f^{\prime}\left(k^{*}\right)-g^{\prime}\left(k^{*}\right)\right\} \\
+u^{\prime \prime}\left(c^{*}\right)\left[\mu f^{\prime}\left(k^{*}\right)-(\mu-1) g^{\prime}\left(k^{*}\right)\right]\left\{f^{\prime \prime}\left(k^{*}\right)-g^{\prime \prime}\left(k^{*}\right)\right\} \\
+u^{\prime}\left(c^{*}\right)\left[\mu f^{\prime \prime \prime}\left(k^{*}\right)-(\mu-1) g^{\prime \prime \prime}\left(k^{*}\right)\right]
\end{array}\right\},
$$

and

$$
T=\left\{\begin{array}{c}
\left.u^{\prime \prime}\left(c^{*}\right)\left[\mu f^{\prime}\left(k^{*}\right)-(\mu-1) g^{\prime}\left(k^{*}\right)\right)\right]\left\{f^{\prime}\left(k^{*}\right)-g^{\prime}\left(k^{*}\right)\right\} \\
+u^{\prime}\left(c^{*}\right)\left[\mu f^{\prime \prime}\left(k^{*}\right)-(\mu-1) g^{\prime \prime}\left(k^{*}\right)\right]
\end{array}\right\} .
$$

From $(\nabla),(\star)$ and $(\diamond), S$ and $T$ are respectively calculated as

$$
\begin{gathered}
S=\frac{1}{\beta\left\{g^{\prime}\left(k^{*}\right)\right\}^{2}}\left[u^{\prime \prime}\left(c^{*}\right)\left\{f^{\prime \prime}\left(k^{*}\right)-f^{\prime}\left(k^{*}\right) \frac{g^{\prime \prime}\left(k^{*}\right)}{g^{\prime}\left(k^{*}\right)}\right\}+u^{\prime \prime \prime}\left(c^{*}\right)\left\{f^{\prime}\left(k^{*}\right)-g^{\prime}\left(k^{*}\right)\right\}^{2}\right], \\
T=\frac{1}{\beta g^{\prime}\left(k^{*}\right)} u^{\prime \prime}\left(c^{*}\right)\left\{f^{\prime}\left(k^{*}\right)-g^{\prime}\left(k^{*}\right)\right\} .
\end{gathered}
$$

By Theorem 4, we already have

$$
\hat{g}_{2}\left(k^{*}, 0\right)=\frac{g^{\prime}\left(k^{*}\right)}{f^{\prime}\left(k^{*}\right)}\left\{h\left(k^{*}\right)+\beta(\mu-1) \frac{u^{\prime}\left(c^{*}\right)}{u^{\prime \prime}\left(c^{*}\right)} h^{\prime}\left(k^{*}\right)\right\} .
$$

Obviously $\tilde{g}_{2}\left(k^{*}, 0\right)=h\left(k^{*}\right), \quad \tilde{g}_{12}\left(k^{*}, 0\right)=h^{\prime}\left(k^{*}\right), \quad \tilde{g}_{112}\left(k^{*}, 0\right)=h^{\prime \prime}\left(k^{*}\right)$. Then plugging all of these into $(\bullet)$, and arranging, again, with $(\star)$ and $(\diamond)$, we derive desired results. 